\title{
Predição do baixo desempenho motor por meio de indicadores antropométricos em crianças de oito a 10 anos
}

https://doi.org/10.11606/issn.1981-4690.v35i2p353-362

\author{
Raísa Carvalho da Silva* \\ Geraldo J ose Ferrari J unior* \\ Fernando Luiz Cardoso* \\ J oão Otacilio Libardoni dos Santos* \\ Érico Pereira Gomes Felden* \\ Thaís Silva Beltrame*
}

*Universidade do Estado de Santa Catarina, Florianópolis, SC, Brasil.

**Universidade Federal do Amazonas, Manaus, AM, Brasil.

\section{Resumo}

Este estudo teve como objetivo analisar a capacidade preditiva e possíveis pontos de corte dos indicadores antropométricos na predição do baixo desempenho motor em ambos os sexos. Participaram do estudo 350 escolares (186 meninas e 164 meninos) com idades de oito a 10 anos. Fizeram parte da pesquisa três escolas, sendo duas escolas públicas e uma escola privada localizadas na cidade de Manaus-AM. Os indicadores antropométricos avaliados foram: Índice de Massa Corporal (IMC), Circunferência de Cintura (CC) e Razão Cintura Estatura (RCEst), e para avaliar as tarefas motoras foram utilizados os instrumentos Movement Assessment Battery for Children - Second Edition (MABC-2) e Körperkoordination Test für Kinder (KTK). A curva Receiver Operating Characteristic (ROC) foi utilizada para estimar a capacidade preditiva dos indicadores antropométricos (IMC, CC e RCEst), apontando o melhor ponto de corte para a predição do baixo desempenho motor. Foi possível verificar capacidade preditiva dos indicadores antropométricos no somatório dos quocientes motores no instrumento KTK na amostra geral $(p<0,001)$ e quando a amostra foi estratificada por sexo, houve capacidade preditiva pelo indicador IMC em meninos e meninas ( $p=0,006$ e $p=0,012$, respectivamente), da mesma forma para o indicador $C C$ ( $p=0,012$ e $p<0,001$, respectivamente). 0 indicador antropométrico RCEst apresentou capacidade preditiva apenas para o sexo feminino $(p<0,001)$. Já no MABC-2 não foram encontradas diferenças significativas. Os indicadores antropométricos (IMC, CC e RCEst) possuem capacidade preditiva em algumas tarefas do KTK, porém não apresentaram predição para o baixo desempenho motor no MABC-2.

Palavras-Chave: Habilidade motora; Pontos de corte; Indicadores antropométricos; Criança.

\section{Introdução}

Odesempenho motor em estudos desenvolvimentistas é investigado a partir de baterias motoras que geralmente avaliam habilidades motoras fundamentais e capacidades físicas e motoras das crianças, como Körperkoordination Test für Kinder (KTK) ${ }^{1}$, Movement Assessment Battery for Children - Second Edition (MABC2) ${ }^{2}$, Test of Gross Motor Development - Second Edition (TGMD-3) ${ }^{3}$ dentre outras. Estudos envolvendo esta questáo $0^{4,5}$, direcionam um crescente número de crianças que apresentam um baixo desempenho motor. Seguindo a perspectiva desenvolvimentista, Gallahue e Ozmun ${ }^{6}$ propõem que a interação entre a tarefa (motora) com os fatores ambientais e individuais podem influenciar como é expressado a habilidade na criança, ou seja, esses fatores influenciam determinantemente para um bom desempenho ou um baixo desempenho motor.

Ainda, estudos de revisão apontam que indivíduos ativos são mais competentes motoramente, quando se trata de habilidades motoras fundamentais ${ }^{7}$. Sendo 
o contrário também verdadeiro, ou seja, a pouca adesão na prática de atividades físicas das crianças pode associar-se aos baixos níveis de desempenho motor8. Este baixo desempenho motor relatado pode ser mediado pelo número de experiências vividas, fator considerável para aquisição de novas habilidades motoras'. Outro agravante é o aumento as prevalências de sobrepeso e obesidade que está associado ao baixo nível de atividade física ${ }^{10} \mathrm{e}$ está associada às dificuldades motoras, principalmente quanto a locomoção?

Esta relaçáo entre baixo desempenho motor e excesso de peso tende a ser um ciclo, no qual as crianças que apresentam alguma dificuldade motora tende a adotar um estilo mais sedentário e fuga das atividades que não faz tão satisfatoriamente e consequentemente pode

\section{Método}

\section{Participantes do estudo}

Participaram do estudo 350 escolares com idades de oito a 10 anos, de ambos os sexos. Fizeram parte desta pesquisa três escolas, sendo duas escolas públicas e uma escola privada, selecionadas de acordo com a disponibilidade de participação das mesmas, localizadas na cidade de Manaus-AM. A amostragem foi do tipo não probabilística intencional, pois não utilizou fórmulas e critérios de equiprobabilidade, sendo constituída pelos escolares que demonstraram interesse em participar do estudo.

Foram considerados como critérios de inclusão os seguintes aspectos: ter idade entre oito e dez anos; a criança náo deve apresentar, a partir de dados informados pelos professores e equipe diretiva da escola, deficiências físicas e/ou mentais, sintomas de dor e desconforto ou ainda ter sido submetido à cirurgia traumatortopédicas em membros superiores e inferiores.

Os pais das crianças foram informados do procedimento da pesquisa e assinaram o Termo de Consentimento Livre e Esclarecido - TCLE, autorizando assim a criança a participar. $\mathrm{O}$ estudo foi aprovado pelo Comitê de Comitê de Ética e Pesquisa em Seres Humanos da Universidade do Estado de Santa Catarina (UDESC), sob o parecer no $159.196 / 2012$ respeitando os preceitos da experimentação com seres humanos, todos os participantes permaneceram anônimos. ocasionar um aumento de peso ${ }^{8}$.

Tendo em vista que para avaliar status de peso das crianças, o método mais eficaz de mensuração e o mais viável para os professores de educação física escolar, são os indicadores antropométricos. Assim, aliar uma nova função para estes indicadores, no caso, identificar crianças com baixo desempenho motor a partir destes os indicadores antropométricos torna-se uma ferramenta útil e acessível, diferentemente das baterias motoras que por questôes financeiras, não são acessíveis às escolas públicas. Neste ínterim, o presente estudo teve como objetivo analisar a capacidade preditiva e possíveis pontos de corte dos indicadores antropométricos na predição do baixo desempenho motor em ambos os sexos.

\section{Variáveis do estudo}

As tarefas motoras utilizadas na pesquisa foram provenientes dos instrumentos Movement Assessment Battery for Children - Second Edition (MABC-2) proposto por Henderson, Sugden e BARNETT ${ }^{2}$ e Körperkoordination Test für Kinder (KTK) proposto por Kiphard e Schilling ${ }^{1}$. A seleção dos instrumentos levou em conta o número de pesquisas científicas e a relevância no cenário nacional e internacional ${ }^{5,8,11-17}$. Portanto, o uso de tal tarefas levou em consideração a quantidade de estudos com esses instrumentos, no entanto náo foi objetivo do estudo comparar as baterias motoras, visto que foram criadas com propostas diferentes.

O MABC-2 é instrumento validado no Brasil $^{18}$, constituído por três conjuntos de tarefas adequadas para cada faixa etária (faixa $1-3$ a 6 anos; faixa $2-7$ a 10 anos e faixa 3 11 a 16 anos). As tarefas investigadas foram: a) Colocando pinos; b) Passando o cordão; trilha da bicicleta; c) Recebendo com duas mãos; d) Arremessando o saco de feijão no alvo; e) Equilíbrio sobre a tábua; f) Caminhando sobre a linha; g) Saltando em tapetes.

A partir do resultado dos testes, os escores brutos foram transformados em escores padróes, e os escores padróes foram somados dentro de cada habilidade, e entre si, resultando no escore padrão total. $\mathrm{O}$ resultado do escore padrão de cada habilidade e do escore padrão total foi 
comparado, por meio de tabelas, aos percentis do teste. Classificando os sujeitos a partir dos percentis em dificuldade significativa de movimento (valores menores ou iguais ao percentil 5), risco para dificuldade de movimento (valores entre o percentil 5 e 15) e desenvolvimento normal (valores superiores ou iguais ao percentil 16). Para as análises, as classificações foram agrupadas em: dificuldade/risco significativa de movimento e desempenho normal. O agrupamento foi uma opção metodológica alinhando-se ao objetivo do presente estudo que foi propor um ponto de corte para o baixo desempenho motor, ou seja, foi necessário o agrupamento dessas categorias, visto que em ambas categorias essas crianças já apresentavam de alguma forma dificuldades no desempenho.

O KTK abrange quatro testes: a) Equilíbrio em marcha à retaguarda; b) Saltos monopedais; c) Saltos laterais; d) Transposição lateral. A bateria motora engloba crianças e adolescentes de 5 a 14 anos de idade. Após a aplicação dos testes, o resultado de cada tarefa foi comparado com os valores normativos (tabelas elaboradas pelos autores do KTK). Para cada tarefa do teste é atribuído um quociente motor (QM), e a soma dos quatro quocientes motores gera o somatório dos quocientes motores (SQM), este pode ser apresentado percentil e em valores absolutos. A partir desses valores as crianças foram classificadas conforme sexo e idade nas seguintes categorias: (1) perturbaçóes da coordenação (QM < 70); (2) insuficiência coordenativa (71 $\leq \mathrm{QM} \leq$ 85); (3) coordenação normal ( $86 \leq \mathrm{QM} \leq 115)$; (4) coordenação boa $(116 \leq \mathrm{QM} \leq 130)$; (5) coordenação muito boa $(131 \leq \mathrm{QM} \leq 145)$. Para as análises da pesquisa, as classificaçóes gerais foram agrupadas em: Perturbaçóes/insuficiência coordenativa e coordenação normal (coordenação normal/boa/ muita boa) os autores do instrumento avaliam a coordenação motora exclusivamente com valores médios, a partir disso para classificar as crianças nas tarefas, foi dividida a pontuação do QM em tercis e classificada em desempenho normal (66,6 e 99,9\%), e desempenho abaixo do esperado (33,3\%).

Para a obtenção dos dados antropométricos os procedimentos foram padronizados seguindo a orientação de Alvarez e PAVAN ${ }^{19}$, as crianças usaram roupas adequadas para a prática de Educação Física, e não utilizavam calçados. Para a obtenção dos valores de massa corporal, foi utilizada uma balança digital da marca LAICA, com plataforma, escalonada em quilos e intervalos de 200 gramas, com registro mínimo de $12 \mathrm{~kg}$ e máximo de $136 \mathrm{~kg}$.
A estatura foi mensurada utilizando uma fita métrica com escala de $0,1 \mathrm{~cm}$, fixada a parede sem rodapé, o aluno foi posicionado em pé com o peso igualmente distribuído, tomando a posição ortostática.

Para avaliação da circunferência de cintura (CC) foi utilizada uma fita antropométrica em fibra de vidro (Sanny ${ }^{\oplus}$, Brasil) no ponto médio entre a última costela e a crista ilíaca. A partir dos dados antropométricos coletados foi determinado o índice de massa corporal (IMC = massa corporal $(\mathrm{kg}) /$ estatura $\left.^{2}(\mathrm{~m})\right)$. O status de peso foi classificado por meio do IMC, onde os critérios utilizados foram os valores de referência proposto por Cole et al. ${ }^{20}$ e Cole et al. ${ }^{21}$ que classifica em baixo peso, eutrofia, sobrepeso e obeso. A razão cintura-estatura (RCEst) foi determinada pela divisão da circunferência da cintura $(\mathrm{cm})$ pela estatura $(\mathrm{cm})$.

\section{Análise de dados}

A análise dos dados foi feita a partir do programa estatístico Statistical Package for Social Sciences (SPSS) versão 20.0. Para análise descritiva foi utilizado medidas descritivas com médias, desviospadrão e distribuição de frequências. Para verificar a normalidade dos dados foi utilizado o teste de Kolmogorov-Smirnov.

Em relação à estatística inferencial, para a associação das variáveis categóricas: sexo, status de peso e classificação motora foi utilizado o teste de associação Qui-Quadrado. Utilizou-se o U de Mann Whitney para ver as diferenças entre meninos e meninas nas variáveis numéricas: idade, IMC, CC, RCEst, escore padrão final do MABC-2 e do KTK. Para calcular a curva Receiver Operating Characteristic (ROC) foi utilizada a versão free do programa MedCalc, dessa forma estimou-se a capacidade preditiva dos indicadores antropométricos (IMC, CC e RCEst), apontando o melhor ponto de corte para a predição do baixo desempenho motor.

A partir dessa análise, foram apresentados os índices: área sob a curva ROC (AUC), valores de sensibilidade e especificidade e pontos de corte para os indicadores antropométricos. A área sob a curva ROC representa a capacidade do teste de classificar corretamente os participantes com alto ou baixo desempenho motor. Os valores da curva ROC variam entre 1 e 0,5 , onde 1 é um considerado um teste perfeito e abaixo 0,5 um teste inadequado ${ }^{22}$. Foi adotado intervalo $95 \%$ de confiança $(p<0,05)$. 


\section{Resultados}

A amostra geral indicou IMC com média de $17,67 \mathrm{~kg} / \mathrm{m}^{2}$, e quando categorizado a percentagem de crianças obesas foi de 8,0\%. Foi observada diferença significativa entre os sexos no escore padrão final do KTK $(p<0,001)$, sendo o escore dos meninos superior $(p<0,001)$. Nas tarefas de saltos monopedais $(\mathrm{p}<0,001)$, saltos laterais $(\mathrm{p}<0,001)$ e transposiçâo lateral $(\mathrm{p}<0,001)$ o escore dos meninos foram superiores, porém não houve diferença significativa na tarefa de equilíbrio em marcha à retaguarda $(\mathrm{p}=0,674)$. Quanto ao escore padrão final do MABC-2 não houve diferenças significativas. Ainda, verificou-se que $4,9 \%$ dos meninos e $23,1 \%$ das meninas apresentaram perturbaçóes ou insuficiência coordenativa no KTK. Já no MABC, 33,5\% dos meninos e 36,0\% das meninas apresentaram dificuldade ou risco de movimento. Quanto aos indicadores antropométricos (IMC, CC, RCE), não foram encontradas diferenças significativas entre os sexos (TABELA 1).

TABELA 1 - Dados descritivos da amostra.

*p valor do teste $\mathrm{U}$ de Mann - Whitney para as variáveis contínuas e do Qui-quadrado para as variáveis categóricas. Os valores foram expressos em média (desvio-padrão) ou percentuais/QM: Quociente motor das tarefas.

\begin{tabular}{lcccc}
\hline Variável & Geral & Meninos & Meninas & p-valor* \\
\hline Idade, anos & $8,94(0,78)$ & $8,99(0,79)$ & $8,90(0,77)$ & 0,317 \\
IMC, Kg/m2 & $17,67(3,53)$ & $17,57(3,44)$ & $17,76(3,62)$ & 0,658 \\
Status de peso, \% & & & & \\
$\quad$ Abaixo do peso & 10,6 & 8,5 & 12,4 & \\
$\quad$ Eutrófico & 65,1 & 68,9 & 61,8 & 0,519 \\
$\quad$ Sobrepeso & 16,3 & 15,2 & 17,2 & \\
$\quad$ Obeso & 8,0 & 7,3 & 8,6 & \\
Circunferência de cintura, cm & $65,74(10,32)$ & $65,58(10,13)$ & $65,87(10,52)$ & 0,837 \\
Razão cintura estatura (RCEst), cm & $0,48(0,06)$ & $0,48(0,06)$ & $0,48(0,06)$ & 0,697 \\
KTK, escore padrão final & $95,50(12,32)$ & $99,83(9,59)$ & $91,69(13,20)$ & $<0,001$ \\
KTK, \% & & & & \\
$\quad$ Com perturbaçóes e insuficiência coordenativa & 14,6 & 4,9 & 23,1 & $<0,001$ \\
$\quad$ Coordenaçăo normal & 85,4 & 95,1 & 76,9 & \\
Equilíbrio em marcha à retaguarda, QM & $93,72(12,14)$ & $93,29(12,00)$ & $94,10(12,28)$ & 0,674 \\
Saltos monopedais, QM & $60,39(11,63)$ & $64,19(10,93)$ & $57,04(11,21)$ & $<0,001$ \\
Saltos laterais, QM & $76,96(16,18)$ & $84,59(13,51)$ & $70,22(15,35)$ & $<0,001$ \\
Transposiçăo lateral, QM & $75,86(13,74)$ & $79,12(13,20)$ & $72,99(13,59)$ & $<0,001$ \\
MABC, total de pontos & $68,94(11,83)$ & $69,09(11,26)$ & $68,82(12,34)$ & 0,814 \\
MABC, \% & & & & \\
$\quad$ Com dificuldade e risco de movimento & 34,9 & 33,5 & 36,0 & 0,626 \\
$\quad$ Desempenho normal, \% & 65,1 & 66,5 & 64,0 & \\
\hline
\end{tabular}

356 • Rev Bras Educ Fís Esporte, (São Paulo) 2021 Abr-J un;35(2)353-362 
Em relação às análises da curva $\mathrm{ROC} e$ bateria KTK, observou-se que os indicadores antropométricos (IMC, CC e RCEst) obtiveram possível capacidade preditiva para o baixo desempenho motor $(\mathrm{p}<0,001)$, porém quando a amostra foi estratificada por sexo, houve capacidade preditiva pelo indicador IMC em meninos e meninas ( $p=0,006$ e $p=0,012$, respectivamente), da mesma forma para o indicador CC $(\mathrm{p}=0,012$ e $\mathrm{p}<0,001$, respectivamente). Já o indicador antropométrico RCEst apresentou capacidade preditiva apenas para o sexo feminino $(p<0,001)$. Os pontos de corte para os meninos foram IMC de $18,94 \mathrm{~kg} / \mathrm{m}^{2}$ e CC de, $75,8 \mathrm{~cm}$ para IMC e CC, respectivamente e nas análises das meninas os pontos de corte foram $18,06 \mathrm{~kg} / \mathrm{m}^{2}, 64,9 \mathrm{~cm} \mathrm{e}$ $0,51 \mathrm{~cm}$ para IMC, CC e RCEst respectivamente, apresentando os melhores indicadores de sensibilidade e especificidade (TABELA 2).

TABELA 2 - Valores da curva ROCpara o instrumento deavaliação KTK, para cada sexo eindicadorantropométrico.

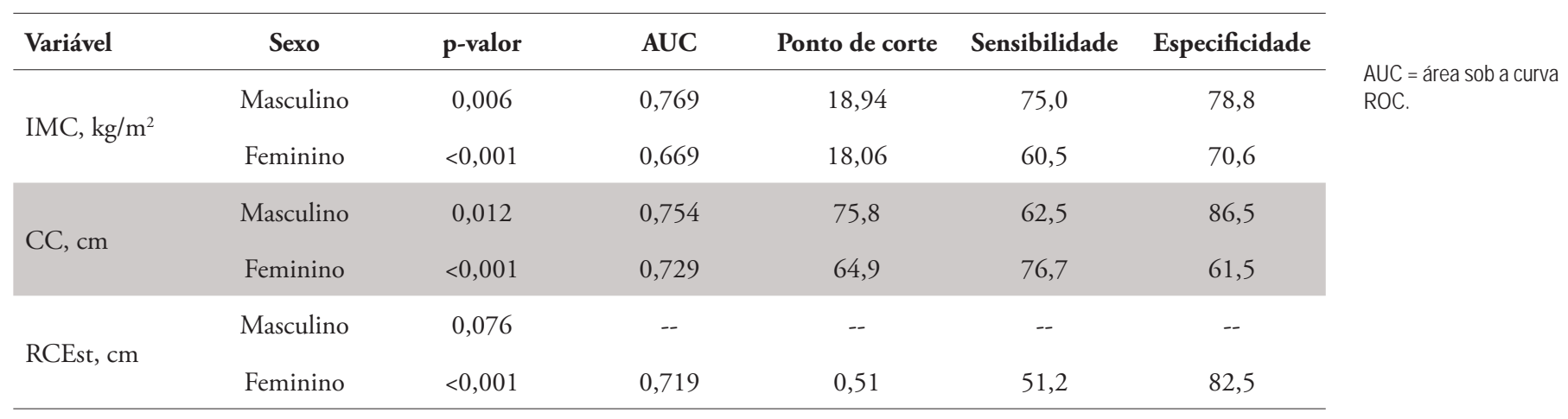

Nas análises da curva ROC nas tarefas do KTK no sexo masculino os indicadores antropométricos são possíveis preditores em apenas duas tarefas (equilíbrio em marcha à retaguarda e saltos monopedais). No IMC na tarefa de equilíbrio em marcha à retaguarda apresentou ponto de corte 18,93 pontos $(\mathrm{p}=0,039)$ e os saltos monopedais apresentaram ponto de corte 18,62 pontos $(\mathrm{p}=0,003)$. Na CC o equilíbrio em marcha à retaguarda teve ponto de corte 71 pontos $(\mathrm{p}<0,001)$ e para a tarefa de saltos monopedais o ponto de corte foi de 74 pontos $(p<0,001)$. Já a RCEst apresentou ponto de corte 0,53 tanto para equilíbrio em marcha à retaguarda $(\mathrm{p}=0,023)$, quanto para saltos monopedais $(\mathrm{p}=0,002)$ (TABELA 3).

Quanto às análises referentes à análise da curva ROC nas tarefas do KTK no sexo feminino, o IMC não apresentou capacidade preditiva na tarefa de saltos laterais, porém apresentou capacidade preditiva nas tarefas equilíbrio em marcha à retaguarda $(\mathrm{p}=0,039)$ e saltos monopedais ( $\mathrm{p}=0,003)$. A CC e RCEst obtiveram capacidade preditivas em todas as tarefas $(p<0,05)$ (TABELA 4).

Por fim, quando executado a busca do ponto de corte para os grupos de tarefas do MABC-2 e para seu escore final, não foi verificado resultado estatisticamente significativo ( $p>0,05)$. Com isso, os resultados não foram apresentados em tabela. 
Silva RC, et al.

TABELA 3 - Análise da curva ROC nas tarefas do KTK no sexo masculino.

AUC = área sob a curva ROC.

\begin{tabular}{|c|c|c|c|c|c|c|}
\hline $\begin{array}{l}\text { Indicadores } \\
\text { Antropométricos }\end{array}$ & $\begin{array}{l}\text { Tarefas do KTK, } \\
\text { em pontos }\end{array}$ & p-valor & AUC & Ponto de corte & Sensibilidade & Especificidade \\
\hline \multirow{4}{*}{$\mathrm{IMC}, \mathrm{kg} / \mathrm{m}^{2}$} & $\begin{array}{l}\text { Equilíbrio em } \\
\text { marcha à retaguarda }\end{array}$ & 0,039 & 0,600 & 18,93 & 43,4 & 84,7 \\
\hline & Saltos monopedais & 0,003 & 0,686 & 18,62 & 60,0 & 78,4 \\
\hline & Saltos laterais & 0,278 & -- & -- & -- & -- \\
\hline & Transposiçăo lateral & 0,503 & -- & -- & -- & -- \\
\hline \multirow{4}{*}{$\mathrm{CC}, \mathrm{cm}$} & $\begin{array}{l}\text { Equilíbrio em } \\
\text { marcha à retaguarda }\end{array}$ & 0,025 & 0,608 & 71 & 39,5 & 82,9 \\
\hline & Saltos monopedais & $<0,001$ & 0,759 & 74 & 56,0 & 89,2 \\
\hline & Saltos laterais & 0,129 & - & -- & -- & -- \\
\hline & Transposiçāo lateral & 0,342 & -- & -- & -- & -- \\
\hline \multirow{4}{*}{ RCEst,cm } & $\begin{array}{l}\text { Equilíbrio em } \\
\text { marcha à retaguarda }\end{array}$ & 0,023 & 0,609 & 0,53 & 34,0 & 86,5 \\
\hline & Saltos monopedais & 0,002 & 0,690 & 0,53 & 56,0 & 86,3 \\
\hline & Saltos laterais & 0,166 & -- & -- & -- & -- \\
\hline & Transposição lateral & 0,532 & -- & -- & -- & -- \\
\hline
\end{tabular}

TABELA 4 - Análise da curva ROC nas tarefas do KTK no sexo feminino.

$\mathrm{AUC}=$ área sob a curva ROC.

Tarefas do KTK

p-valor

AUC

Ponto de corte

Sensibilidade Especificidade

Antropométricos

\begin{tabular}{|c|c|c|c|c|c|c|}
\hline \multirow{3}{*}{$\mathrm{IMC}, \mathrm{Kg} / \mathrm{m}^{2}$} & $\begin{array}{l}\text { Equilíbrio em } \\
\text { marcha à retaguarda }\end{array}$ & 0,001 & 0,657 & 18,35 & 54,0 & 75,6 \\
\hline & Saltos monopedais & 0,001 & 0,637 & 17,67 & 59,5 & 69,9 \\
\hline & Saltos laterais & 0,108 & -- & -- & -- & -- \\
\hline \multirow{2}{*}{$\mathrm{CC}, \mathrm{cm}$} & $\begin{array}{l}\text { Equilíbrio em } \\
\text { marcha à retaguarda }\end{array}$ & $<0,001$ & 0,664 & 61,5 & 81,0 & 49,6 \\
\hline & Saltos monopedais & $<0,001$ & 0,653 & 64,9 & 63,1 & 65,7 \\
\hline \multirow{4}{*}{ RCEst,cm } & $\begin{array}{l}\text { Equilíbrio em } \\
\text { marcha à retaguarda }\end{array}$ & $<0,001$ & 0,663 & 0,46 & 76,2 & 53,7 \\
\hline & Saltos monopedais & 0,033 & 0,590 & 0,47 & 57,1 & 59,8 \\
\hline & Saltos laterais & 0,028 & 0,591 & 0,50 & 43,6 & 76,5 \\
\hline & Transposição lateral & $<0,001$ & 0,666 & 0,47 & 65,3 & 63,2 \\
\hline
\end{tabular}

358 • Rev Bras Educ Fís Esporte, (São Paulo) 2021 Abr-J un;35(2)353-362 


\section{Discussão}

Os principais achados do presente estudo sugerem que, nos resultados do instrumento de avaliação KTK, o IMC e a CC fornecem melhor capacidade preditiva em comparação com o RCEst para os meninos. Nas meninas o IMC mostrou uma precisão discriminatória ligeiramente melhor na prediçáo do baixo desempenho motor em comparação com a CC e RCEst. Em contrapartida, não foram apresentadas capacidades preditivas dos indicadores antropométricos para a pontuação total da bateria motora MABC-2, também como para seus grupos de tarefa. É possível que as diferenças entre as baterias estivessem relacionadas ao tipo de tarefas e níveis de exigência de cada uma delas, no entanto a origem e a causa dessas diferenças não serão discutidas no estudo, visto que este não foi o objetivo do trabalho.

O teste de coordenaçáo corporal KTK consiste em quatro tarefas motoras, essas tarefas são constituídas com a finalidade de avaliar o equilíbrio, ritmo, lateralidade, velocidade, agilidade e força muscular das crianças e adolescentes. De Deus et al. ${ }^{23}$, acompanharam o desempenho de crianças de 6 a 10 anos nos testes do KTK e verificaram que as competências coordenativas exigidas nas tarefas do KTK apresentaram trajetórias diferentes.

A tarefa de saltos laterais segundo Guedes e $\mathrm{NETO}^{24}$ pretende avaliar velocidade/agilidade em saltos alternados, nesta tarefa a criança precisa de rápidas contrações musculares no curto tempo de 15 segundos e mudar a direção do corpo, com isso, é necessário que a cada salto seja restabelecido a posição do centro de massa dentro dos limites da base de apoio, para evitar a queda sendo necessário maior controle do equilíbrio. No estudo de BERLEze et al. $^{25}$ as crianças obesas apresentaram maior dificuldade nesta tarefa quando comparadas com seus pares eutróficos, os autores justificam devido ao comportamento sedentário e a falta de atividades motoras dessas crianças. No presente estudo, dois indicadores antropométricos foram preditores do baixo desempenho motor na tarefa de saltos laterais nas meninas, sendo a CC com o ponto de corte de $64 \mathrm{~cm}$ e a RCEst $0,50 \mathrm{~cm}$.

Segundo GUEDES e NETO ${ }^{24}$ a tarefa de transposição lateral avalia lateralidade e espaço-temporal, exigindo da criança um esquema interno para facilitar o deslocamento sobre as plataformas em um determinado tempo. Nesta pesquisa, os indicadores antropométricos não tiveram capacidade preditiva nesta tarefa para os meninos, porém nas meninas os pontos de corte foram de $16,27 \mathrm{~kg} / \mathrm{m}^{2}$ para o IMC, $62 \mathrm{~cm}$ para CC e $0,47 \mathrm{~cm}$ para RCEst. O fato do ponto de corte dos indicadores antropométricos nessa tarefa ter sido baixo pode ser devido à pouca variabilidade do rendimento das meninas nessa tarefa, náo sendo relevante a adiposidade corporal.

$\mathrm{Na}$ tarefa de saltos monopedais é avaliada coordenação e força de membros inferiores, no estudo de Lemos et al. ${ }^{26}$ foi verificado que as crianças apresentaram maior dificuldade nessa tarefa e na de equilíbrio em marcha a retaguarda. A tarefa de equilíbrio em marcha a retaguarda esta tem por objetivo avaliar o equilíbrio dinâmico das crianças. O equilíbrio segundo Teixeira et al. ${ }^{27}$, é uma integração entre os sistemas visual, somatossensorial e o vestibular que interagem juntamente com o sistema nervoso central, mais especificamente tronco encefálico e cerebelo para a manutenção do equilíbrio.

Para Luz et al. ${ }^{28}$ a tarefa de equilíbrio em marcha à retaguarda exige deslocamento do centro de gravidade uma forma equilibrada, o que pode penalizar as crianças com maior adiposidade corporal. Para Lemos et al. ${ }^{26}$, o aumento do IMC em indivíduos obesos reduz a habilidade de fazer ajustes posturais, aumentando a instabilidade postural. Lemos et al. ${ }^{26}$, explicam que as capacidades físicas como coordenação, agilidade e força de membros inferiores estão ligadas com a capacidade de controle postural, assim como variáveis antropométricas.

Os indicadores antropométricos, no presente estudo, obtiveram melhor AUC nas duas últimas tarefas citadas, tanto para o sexo feminino, quanto para o sexo masculino. Cabe ressaltar que a circunferência de cintura foi o indicador antropométrico que melhor previu o baixo desempenho motor na tarefa de saltos monopedais, este fato pode ser devido à exigência de força na execução da mesma, como explicado por Berleze et al..$^{25}$ crianças com sobrepeso e obesidade apresentam piores desempenhos nas tarefas que envolvem força e resistência aeróbica quando comparadas com crianças eutróficas.

No presente estudo, crianças com IMC de $18,7 \mathrm{~kg} / \mathrm{m}^{2}, 64,9 \mathrm{~cm}$ de CC e $0,53 \mathrm{~cm}$ de RCEst tendem a apresentar piores resultados na bateria do KTK, corroborando com esses achados LOPES et al. ${ }^{22}$ recomendaram para crianças de 9 a 12 anos pontos de corte para IMC, CC e RCEst próximo ao proposto na presente pesquisa. Em um estudo 
de corte transversal com 954 crianças de 5 a 12 anos, D'Hondt et al. ${ }^{29}$ relacionaram IMC e nível de coordenaçáo motora e encontraram que menos de $20 \%$ dos participantes com peso saudável foi identificado com coordenação motora prejudicada, nas crianças com sobrepeso e obesidade a proporção para $43,3 \%$ e até $70,8 \%$, respectivamente.

Por meio de revisão sistemática Lubans et al. ${ }^{30}$ encontraram associaçóes positivas entre coordenação motora e aspectos físicos, psicológicos e função cardiorrespiratória e associação negativa com o status de peso em crianças e adolescentes. Berleze et al..$^{25}$ encontraram que um maior IMC influencia negativamente o processo e o produto no desempenho das habilidades motoras em crianças.

Com base nos resultados encontrados, concluise que os indicadores antropométricos (IMC, CC, RCEst) possuem capacidade preditiva para o baixo desempenho motor a partir de pontos de corte com melhor sensibilidade e especificidade na bateria KTK em ambos os sexos. No entanto quando avaliada para o MABC-2, os indicadores antropométricos não apresentaram capacidade preditiva. A fácil mensuração dos indicadores antropométricos e a acessibilidade para os professores de educação física atuantes na educação básica torna-os ferramentas importantes na avaliação de crianças entre oito e 10 anos. Desta forma, a atual pesquisa possui implicaçóes práticas em âmbito social e científico, fornecendo uma nova possibilidade de mensuração indireta que dê indícios do desempenho motor de crianças. Esta pesquisa limita-se por não ter realizado cálculo amostral, portanto é importante ressaltar que os pontos de corte encontrados no estudo são referentes apenas a essa amostra, tornase necessário novos estudos com um número maior de participantes para que possa generalizar esses resultados. Além disso, deve-se interpretar os pontos de corte com cautela, pois a categorização do baixo desempenho motor pode incluir crianças com dificuldades graves e crianças com um pouco de dificuldade, da mesma forma que o MABC-2 apresenta uma linha tênue entre as classificaçóes de risco de atrasos motores e as dificuldades motoras graves. Apesar disso, uma nova possibilidade de investigação é constituída com a atual pesquisa.

\begin{abstract}
Predictive capacity of anthropometric indicators to indicate low motor performance in children from eight to ten years of age

This study aimed to analyze the predictive capacity and possible cut-off points of anthropometric indicators in the lower motor performance prediction in both sexes. The study included 350 students ( 186 girls and 164 boys) from eight to 10 years old. Three schools participated in the survey, two public schools and a private school located in the city of Manaus, AM. The evaluated anthropometric indicators were: Body Mass Index (BMI), waist circumference (WC), waist Height Ratio (WHtR) and, to evaluate the motor tasks, it was used the Movement Assessment Battery for Children - Second Edition (MABC-2) and Körperkoordination Test für Kinder (KTK). The Receiver Operating Characteristic (ROC) curve was used to estimate the predictive capacity of anthropometric indicators (BMI, WC and WHtR), pointing out the best cut-off point for the low motor performance prediction. It was possible to note predictive capacity of anthropometric indicators in KTK battery in the overall sample $(p<0.001)$ and, when the sample was stratified by sex, there was predictive capacity by $B M I$ indicator in boys and girls ( $p=0,006$ and $p=0,012$, respectively), in the same way as the WC indicator ( $p=0,012$ and $p<0,001$, respectively). And the anthropometric indicator WHtR presented predictive capacity only for girls $(p<0.001)$. In MABC-2, no significant differences were found. The anthropometric indicators (BMI, WC, WHtR) have predictive capacity in some KTK tasks, but they did not show prediction for the low motor performance in MABC-2.
\end{abstract}

KEYwORDS: Motor skill; Cutoff points; Anthropometric indicators; Children. 


\section{Referências}

1. Kiphard E, Schilling F. Body coordination test for children. Weinheim: Beltz Test GmbH; 2007.

2. Henderson SE, Sugden DA, Barnett AL. Movement assessment battery for children: Psychological Corporation London; 1992.

3. Ulrich DA. The test of gross motor development-3 (TGMD-3): Administration, scoring, and international norms. Spor Bilimleri Dergisi. 2013;24(2):27-33.

4. D’Hondt E, Deforche B, Gentier I, De Bourdeaudhuij I, Vaeyens R, Philippaerts R, et al. A longitudinal analysis of gross motor coordination in overweight and obese children versus normal-weight peers. Intern J Obesity. 2013;37(1):61.

5. Jarus T, Lourie-Gelberg Y, Engel-Yeger B, Bart O. Participation patterns of school-aged children with and without DCD. Res Develop Dis. 2011;32(4):1323-31.

6. Gallahue DL, Ozmun JC, Goodway JD. Compreendendo o desenvolvimento motor: bebês, crianças, adolescentes e adultos: AMGH Editora; 2013.

7. de Souza MS, Spessato BC, Valentini NC. Habilidades motoras fundamentais e as possíveis relaçóes com níveis de atividade física, estado nutricional e sexo. ACTA Bras Mov Hum. 2014;4(1):41-51.

8. Santos MMdA, Ribeiro SML, Pellegrini AM, Rocha PRH, Hiraga CY. Crianças com dificuldades motoras apresentam baixos níveis de aptidão física? Motriz. 2012:748-56.

9. Zanella LW, Bandeira PFR, de Souza MS, Valentini NC. Há associação entre o desempenho motor e estado nutricional de escolares? Conexóes. 2015;13(2):136-48.

10. Souza MSd, Spessato BC, Valentini NC. Percepçáo de competência motora e índice de massa corporal influenciam os níveis de atividade física. Rev Bras Cienc Mov. 2014;22(2):78-86.

11. Engel-Yeger B, Hanna Kasis A. The relationship between developmental co-ordination disorders, child's perceived self-efficacy and preference to participate in daily activities. Child Care Health Develop. 2010;36(5):670-7.

12. Liu T, Breslin CM. The effect of a picture activity schedule on performance of the MABC-2 for children with autism spectrum disorder. Res Quarterly Exerc Sport. 2013;84(2):206-12.

13. da Silva J, Beltrame TS. Indicativo de transtorno do desenvolvimento da coordenaçáo de escolares com idade entre 7 e 10 anos. Rev Bras Ciênc Esporte. 2012;35(1).

14. Silman A, Cairney J, Hay J, Klentrou P, Faught BE. Role of physical activity and perceived adequacy on peak aerobic power in children with developmental coordination disorder. Hum Mov Sci. 2011;30(3):672-81.

15. D’Hondt E, Deforche B, Gentier I, Verstuyf J, Vaeyens R, Bourdeaudhuij I, et al. A longitudinal study of gross motor coordination and weight status in children. Obesity. 2014;22(6):1505-11.

16. Melo MM, Lopes VP. Associação entre o índice de massa corporal e a coordenação motora em crianças. Rev Bras Educ Fís Esporte. 2013:7-13.

17. Martins D, Maia J, Seabra A, Garganta R, Lopes V, Katzmarzyk P, et al. Correlates of changes in BMI of children from the Azores islands. Int J Obesity. 2010;34(10):1487.

18. Valentini N, Ramalho M, Oliveira M. Movement Assessment Battery for Children-2: Translation, reliability, and validity for Brazilian children. Res Develop Dis. 2014;35(3):733-40.

19. Alvarez BR, Pavan AL, Petroski E. Alturas e comprimentos. Antropometria. 2003;2:59-71.

20. Cole TJ, Bellizzi MC, Flegal KM, Dietz WH. Establishing a standard definition for child overweight and obesity worldwide: international survey. BMJ. 2000;320(7244):1240.

21. Cole TJ, Flegal KM, Nicholls D, Jackson AA. Body mass index cut offs to define thinness in children and adolescents: international survey. BMJ. 2007;335(7612):194.

22. Lopes L, Santos R, Moreira C, Pereira B, Lopes VP. Sensitivity and specificity of different measures of adiposity to distinguish between low/high motor coordination. J Pediatria. 2015;91(1):44-51.

23. de Deus RKBC, Bustamante A, Lopes VP, Teixeira A, da Silva RMG, Maia JAR. Modelação longitudinal dos níveis de coordenação motora de crianças dos seis aos 10 anos de idade da Região Autônoma dos Açores, Portugal. Rev Bras Educ Fís Esporte. 2010;24(2):259-73.

24. Guedes MS, Neto JLC. Avaliação da coordenação motora em crianças e adolescentes com deficiência auditiva: uma revisão sistemática de estudos brasileiros. Conexôes. 2015;13(3):114-30.

25. Berleze A, Haeffner LSB, Valentini NC. Desempenho motor de crianças obesas: uma investigação do processo e produto de habilidades motoras fundamentais. Rev Bras Cineantropom Desempenho Hum. 2007;9(2):134-44.

26. Lemos LFC, de David AC, Mota CB. Equilíbrio postural: correlaçóes com desempenho motor e variáveis antropométricas 
em crianças de 4 a 10 anos de idade. Saúde Des Hum. 2016;4(1):27-36.

27. Teixeira CS, Alves RF, Pedroso FS. Sistema estomatognático postura e equilíbrio corporal. Salusvita. 2010;29(2):69-81.

28. Luz LG, Seabra A, Padez C, Duarte JP, Rebelo-Gonçalves R, Valente-dos-Santos J, et al. Perímetro de cintura como mediador da influência da maturação biológica no desempenho de coordenação motora em crianças. Rev Paulista Pediatria. 2016;34(3):352-8.

29. D'Hondt E, Deforche B, De Bourdeaudhuij I, Lenoir M. Childhood obesity affects fine motor skill performance under different postural constraints. Neurosci Lett. 2008;440(1):72-5.

30. Lubans DR, Morgan PJ, Cliff DP, Barnett LM, Okely AD. Fundamental movement skills in children and adolescents. Sports Med. 2010;40(12):1019-35.

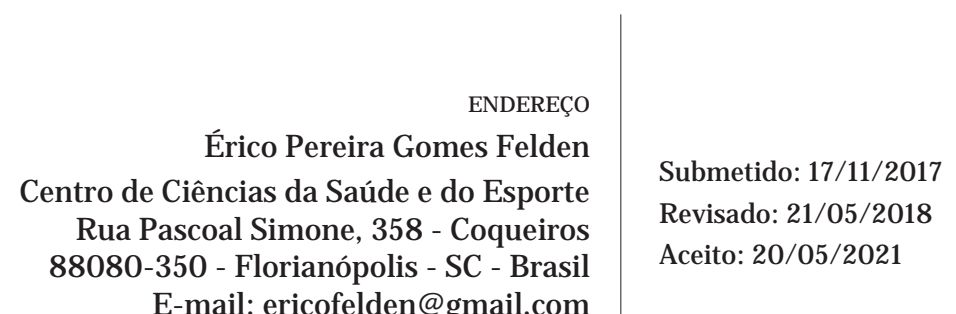

E-mail: ericofelden@gmail.com 